\title{
Enabling vehicular mobility in city-wide IEEE 802.11 networks through predictive handovers
}

\author{
M. Mouton*, G. Castignani, R. Frank, T. Engel \\ Interdisciplinary Centre for Security, Reliability and Trust, University of Luxembourg, 4 rue Alphonse Weicker, L-2721 Luxembourg
}

\section{A R T I C L E I N F O}

\section{Article history:}

Received 22 October 2014

Received in revised form 10 February 2015

Accepted 13 February 2015

Available online 5 March 2015

\section{Keywords:}

IEEE 802.11

Vehicular communications

Handover optimization

Predictive handover

Context-awareness

Experimental evaluation

\begin{abstract}
A B S T R A C T
The increasing number of IEEE 802.11 networks deployed worldwide gives mobile users the possibility of experiencing high-speed wireless access on the move. Moreover, the high density of these deployments in urban areas make IEEE 802.11 a suitable access technology for moving vehicles. However, in order to provide a seamless access to vehicles, the transition between Access Points (APs) must be quick and reliable. The main bottleneck of existing handover mechanisms is the long AP scanning process, which only provides a snapshot of the available networks at a given location, impacting the handover decision on moving vehicles. To overcome this limitation, we propose COPER, a context-based predictive handover mechanism that considers vehicle's trajectory, road topology, and network deployment information to decide the best handover location and candidate access points. We validate with real experiments in a city-wide 802.11 network and show that COPER can provide better average signal strength, data rate, and connected time than other existing handover approaches.
\end{abstract}

(c) 2015 Elsevier Inc. All rights reserved.

\section{Introduction}

In the recent years, the number of mobile users connected through 802.11 networks have significantly increased. At the same time, the increasing number of wireless network deployments open the way towards ubiquitous network connectivity. However, under vehicular mobility, the selection of the AP in providing the most sustainable network connectivity is a challenging task. In this context, the standard IEEE 802.11 handover comprises three phases: scanning, authentication and, association, and is inefficient in ensuring a seamless transition to the best AP. It is well known that the scanning process in which the Mobile Station (MS) probes nearby APs on each channel and waits for probe responses, is the major bottleneck [1-3]. This inefficiency is mainly caused (i) by the long delay in the handover and, (ii) by the lack of indication on short-term changing in the nearby APs' Received Signal Strengths (RSS). The first implies long disconnection periods, while the second leads to potentially inconsistent AP selection.

Many solutions have been proposed to mitigate these issues with some success, mostly by reducing the disconnection period during the handover. The future emergence of IEEE 802.11p/WAVE

\footnotetext{
* Corresponding author. Tel.: +3524666445877.

E-mail addresses: maximilien.mouton@uni.lu (M. Mouton), german.castignani@uni.lu (G. Castignani), raphael.frank@uni.lu (R. Frank), thomas.engel@uni.lu (T. Engel).
}

networks [4], which have been specifically designed for vehicular communications, opens the way towards seamless handovers. One of the major modifications proposed in this standard is the suppression of the authentication and association phase, and the transmission of Wireless Access in Vehicular Environments (WAVE) beacons containing the service transmission characteristics over a dedicated channel. However, this standard still provides no guidance as to how AP selection may be optimized.

As a result, in current and future 802.11 deployments, the inconsistency of the AP selection remains an open issue. Currently, the majority of the proposed handover optimization only rely on the instantaneous sensing of the RSS. However, this metric only provides a snapshot of the current state of the network and does not provide any trend for its evolution, which is critical in the context of vehicular communications. Gustafsson and Jonsson [5] distinguish session continuity as one of the mobility management enhancement that are necessary to achieve the Always Best Connected $(\mathrm{ABC})$ paradigm. The MS needs to augment its knowledge on nearby networks and vehicle's mobility such that it can anticipate their evolution. This anticipation allows the MS to avoid abrupt connection disruption due to inappropriate AP selection and to provide a seamless connection to the AP.

In this paper, we propose COPER, a COntext-aware Predictive handovER technique conceived with the aim of optimizing vehicular communications through a metropolitan IEEE 802.11 hotspot network. The AP selection process of COPER uses a prediction of 
MS connectivity over the near future such that the roaming decision is performed at the most appropriate location. In this case, a prior knowledge of the route taken by the vehicle would allow computing optimal handover locations. However, most of the time, people drive on previously known routes, thus, do not need to be assisted by any embedded system. As a result, there is no way to know the destination, nor the route the driver plans to take in advance. In fact, this information is not reliable since the driver may change the route on impulse. Consequently, COPER includes a direction detection module that provides a short-term prediction of the direction of the vehicle. In order to perform the direction detection and the AP selection, COPER uses the knowledge of the road topology, the APs' locations and their modeled RSS, all stored in a Context DataBase (CDB).

The remainder of this paper is organized as follows. In Section 2 we present related work. Section 3 describes the different modules composing COPER and the CDB construction. The results of the evaluation study are presented in Section 4. Section 5 proposes a discussion on the possible enhancements of coPER. Finally, in Section 6 we conclude the paper.

\section{Related works}

During vehicular mobility, an MS connected through 802.11 networks faces frequent handovers and must select the next AP and associate within the shortest delay. Prior studies on the standard IEEE 802.11 handover mechanism [1-3] identified the scanning phase as the most costly in time. Indeed, during scanning, the MS probes nearby APs and waits for responses on all available channels. Scanning implies a disconnection period because the MS cannot exchange frames with the current AP while listening on other channels. Several approaches have been proposed to reduce or even eliminate the impact of the scanning phase during handover. Montavont et al. [6] proposed an optimization to this process by performing short interleaved scanning phases with on-going data communication. In SyncScan [7], Ramani and Savage investigated a modification of the infrastructure where the APs synchronously broadcast beacon frames on the same channel so that the MS just needs to switch the channel and wait for a short period to retrieve the available APs. Another solution consists in relocating the scanning to a second interface. The experiments conducted by Ramachandran et al. [8] and Brik et al. [9] show that such a solution can provide a significant gain in terms of the time that the MS is associated. In our prior work [10], we evaluated the impact of adding a second radio and showed that the MS can reach up to $98 \%$ of layer 2 connection time. Even though the impact of scanning on handover duration can be significantly reduced, it still suffers from providing inaccurate results. Indeed, scanning is an instantaneous sensing of the network that does not always reflect reality. Because of beacon loss, scanning can miss an available AP, especially in dense deployments, like those discuses in $[11,12]$. To overcome this issue, a solution is to use repeated multiple scans, split into short interleaved scanning phases as described in [6]. However, this approach is not applicable to vehicular communications, as the sensed values become rapidly obsolete because of the relatively high velocity.

In order to provide a dynamic knowledge of nearby APs, Mhatre and Papagiannaki [13] propose performing RSS predictions based on smoothed RSS trends. A similar approach is proposed by Sadiq et al. [14]. Nevertheless, this kind of prediction can only be performed over a very short period, since it does not consider any information on the vehicle's motion. For instance, the MS will not be able to predict a sudden signal decrease when the vehicle leaves the line of sight of the AP.

In the literature, multiple $802.11 \mathrm{p}$ network architectures for Vehicle-to-Infrastructure (V2I) communications have been pro- posed, including multiple handover mechanisms. The network architecture described in [15] consists of IEEE 802.11f Inter Access Point Protocol (IAPP) compatible RoadSide Units (RSU) interconnected by a set of layer 2 switches connected to a gateway router. This makes, the network appear as a single distribution system and mobility management is handled at the layer 2, avoiding the use of Internet Protocol (IP) mobility protocol such as Mobile IPv6 (MIPv6) or Proxy Mobile IPv6 (PMIPv6). The handover is triggered when the On-Board Unit (OBU) sends an IEEE 802.11 disassociation message to the current RSU. The RSU, then, communicates the OBU address to the next RSU by forwarding the packets addressed to OBU. The major drawback of this approach is that it is designed to work in a highway environment where the next RSU selection is trivial. Note that in an urban scenario, the AP selection process may not be as trivial as in the highway scenario. An extended version of this approach presented in [16], is intended to work in an urban environment. The authors propose forwarding data packets to all the candidate RSUs. When an RSU detects that the OBU has entered in its coverage range, it is selected as the next RSU and sends a Move-Notify message to the remaining RSUs. However such an approach implies significant packet overhead.

In order to allow the MS to anticipate the short-term evolution of the network, it is critical to provide information about the vehicle's mobility. Based on the fact that people usually drive on previously known routes, Deshpande et al. [17] propose replacing the scanning with an AP selection using historical information. While driving, the MS learns and caches information about nearby APs (ESSID, BSSID, channel) by frequently sensing during inactive periods. This information is, then, used to script the handover location in advance such that the MS is always connected to the best candidate AP. Another approach is proposed by Kwak et al. [18], who suggest including the trajectory of the vehicle and neighbor information in order to select APs along the vehicle's path and predict an optimal handover location. This approach was investigated by Montavont and Noel [19], who propose an augmented version of the Mobile IPv6 (MIP6) architecture with a new component, a GPS server which is periodically updated with the location of the MS. Based on the evolution of the MS location and prior knowledge of AP locations, channels, BSSIDs and IPv6 prefixes, the GPS server triggers handover to the closest AP when the MS is about to leave the coverage range of the current AP by sending a handover indication packet to the MS. In [20] and [21] the authors use large datasets gathering mobility history of cellular network users [20] and large indoor 802.11 network [21]. Zhang et al. [20] propose a cloud-based mobility prediction system that consists in detecting periodicity in the mobility pattern using the Kullback-Leibler divergence (KLD) as the metric and evaluate social interplay in order to identify some pairs of calls that will be co-located. In [21] Abu-Ghazaleh and Alfa model the mobility behavior of users in a 802.11 network as Markov Renewal Processe (MRP). This model intends to predict handovers and the sojourn duration of a user based on the current location and the prior location of the user immediately before the transition into the current location. In the same way, De Rango et al. [22] proposed an efficient prediction-based bandwidth allocation scheme using the Mobile ReSerVation Protocol (MRSVP) and an analysis of users' mobility to ensure quality-of-service (QoS). These approaches propose long-term predictions that allow the MS to ensure service continuity in case the selected AP is unavailable (switch off or not able to provide enough bandwidth). In our previous contribution (MROAD) [23], the AP selection process uses prior knowledge of the route of the vehicle and the location of the APs along the road. The RSSs of the APs are modeled offline using the CORNER signal propagation model [24]. Based on this information, MROAD can predict the best handover location. This allows the MS to trigger the handover using its location as the single input. 


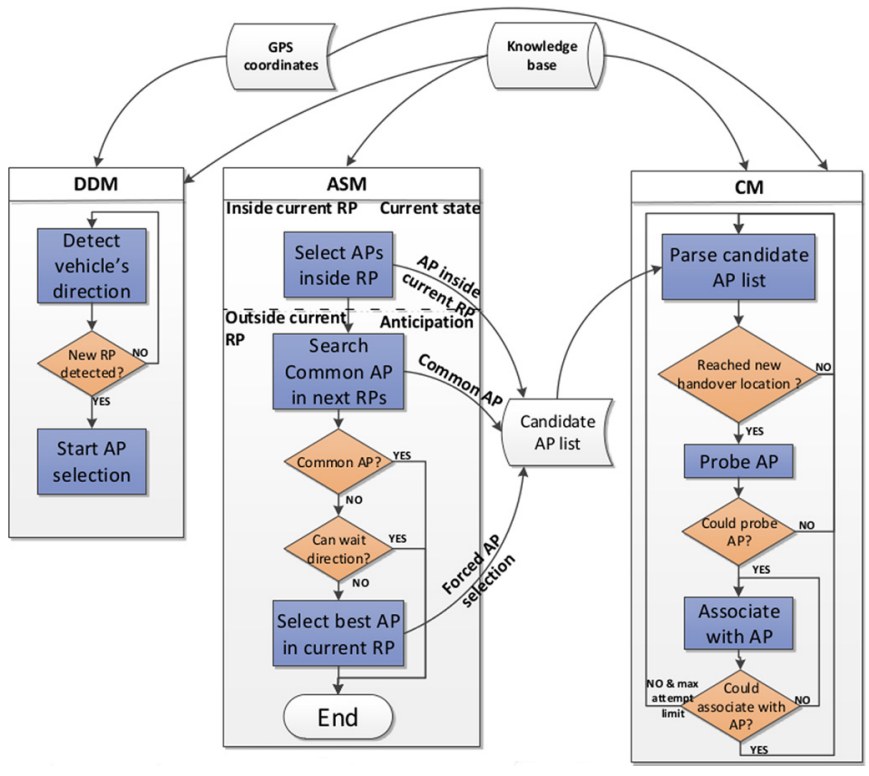

Fig. 1. Description of the three main modules of COPER.

In this paper we intend to select the best AP and predict the handover location without knowing the route of the vehicle in advance. The two main improvements provided by this solution are the addition of a direction detection module, and an AP selection module both relying on both the current location of the vehicle and a CDB of the road topology and the modeled AP RSS.

\section{COPER}

In order to mitigate the limitations of the standard 802.11 handover in a vehicular scenario, we propose a roaming decision technique that intends to provide always best connected wireless access by avoiding the MS scanning process. The choice of the AP providing the best connectivity has traditionally been done by selecting the AP with the highest RSS discovered through the scanning process. In this work, we propose to base the AP selection process on prior knowledge of the road topology, the locations of the APs and a model of their RSSs. Using this information, the MS is able to determine its location on the road network and use the modeled RSSs of nearby APs to choose the one potentially providing the best signal in the new vehicle location.

As illustrated in Fig. 1, COPER is composed of three main modules intended to provide seamless transitions between the best candidate APs regardless of the vehicle's route. To this end, the Direction Detection Module (DDM) anticipates the direction of the vehicle using a fuzzy logic based analysis trajectory that considers the road characteristics stored in a pre-computed CDB. For this direction prediction, the AP Selection Module (ASM) makes use of the simulated AP RSS stored in the CDB in order to look for the best APs and the best locations to trigger handovers. Finally, the Connection Module (CM) detects when the vehicle is entering a handover area and attempts a connection by probing the selected $\mathrm{AP}$ and associating with it. The following sections describe the construction of the CDB and the COPER modules.

\section{1. $C D B$ construction}

COPER decisions are based on the road topology and the modeled RSSs that are stored in a CDB. To build this CDB, we need to gather multiple publicly-available sources of information and organize them in the proposed data structure. In this work we use two notions to characterize the road topology. The first is the Road
Portion (RP) defined as the portion of road bounded by two intersections or a dead end. An RP is characterized by an azimuth $\alpha$ and the coordinates of the starting point $D$ and the ending point $E$. The second notion is the Road Segment (RS), which is a fragment of the RP. Each RP is composed of a set of RSs having equal size (around $5 \mathrm{~m}$ ). Note that both RP and RS are oriented, meaning that a twoway street is composed of two RPs. The CDB structure consists of five tables. Three describe the road topology: the first lists the RPs including their boundaries (in terms of GPS coordinates), size and their RSs; the second lists the RSs including their boundaries, size and the RP they belong to; and the third lists the relations between RPs. The AP table stores the APs' locations, SSIDs, frequencies and the RP each belongs to. Finally, the last table contains the RSSs of the listed APs on each RS obtained through simulation.

The road topology is extracted from the OpenStreetMap ${ }^{1}$ database. The OpenStreetMap web interface provides links for downloading the detailed map of the area selected by the user. This map not only includes the road topology but also a list of Points of Interest (PoI) and a description of the urban topology (e.g., buildings, parks, pedestrian areas). The road topology and the urban topology are extracted and stored in two different files. The road topology is converted into a set of RSs and RPs and stored in the CDB. In addition, we obtain the list of the APs and their locations from an open database provided by the local administration which we include in the CDB. The databased contains a name and the GPS coordinates for each AP of the municipal network. It is used to feed a website mapping different facilities provided by the municipality. The complete list of SSIDs was provided by the network operator. The road topology, AP location and urban topology are provided as an input for the simulation framework.

First, we use sumo [25], an urban mobility simulator, to build a simple mobility scenario where a vehicle drives along all the RPs of the CDB. This scenario is exported to the Qualnet [26] network simulator using the Vergilius framework [27]. The RSSs are computed using CORNER [24], an urban signal propagation model based on knowledge of the road topology. CORNER computes signal attenuation considering three situations: (i) the AP is in the Line of Sight (LoS) of the MS; (ii) the AP is out of the LoS of the MS with one-corner separation; or (iii) the AP is out of the LoS of the MS with two-corner separation. CORNER provides a good trade-off between accuracy and computational cost. In addition to CORNER, Qualnet is set to consider the urban topology in the computation of the signal propagation, in order to provide much more realistic RSS simulations.

A partial visualization of the $\mathrm{CDB}$ is shown in Fig. 2. Based on signal propagation modeling, the $\mathrm{CDB}$ can provide information about the number of APs broadcasting with a minimum required RSS ( $-85 \mathrm{dBm}$ in Fig. 2).

\subsection{Direction detection module}

The DDM attempts to predict the RP the vehicle is about to enter. This module relies on GPS location updates and the road topology provided by the $\mathrm{CDB}$. This information is critical to trigger the handover at the right location, in particular, when the vehicle turns at an intersection and is about to leave the line of sight of the current AP. This situation often leads to a significant decrease of the RSS of the current AP, causing packet loss or even complete disconnection. The DDM distinguishes between the cases of a vehicle continues straight on or turning in an intersection. The first case must correspond to only one candidate RP. Therefore, we consider that the vehicle continues straight on from the current RP to a candidate RP, if the azimuth difference between

\footnotetext{
1 http://openstreetmap.org.
} 


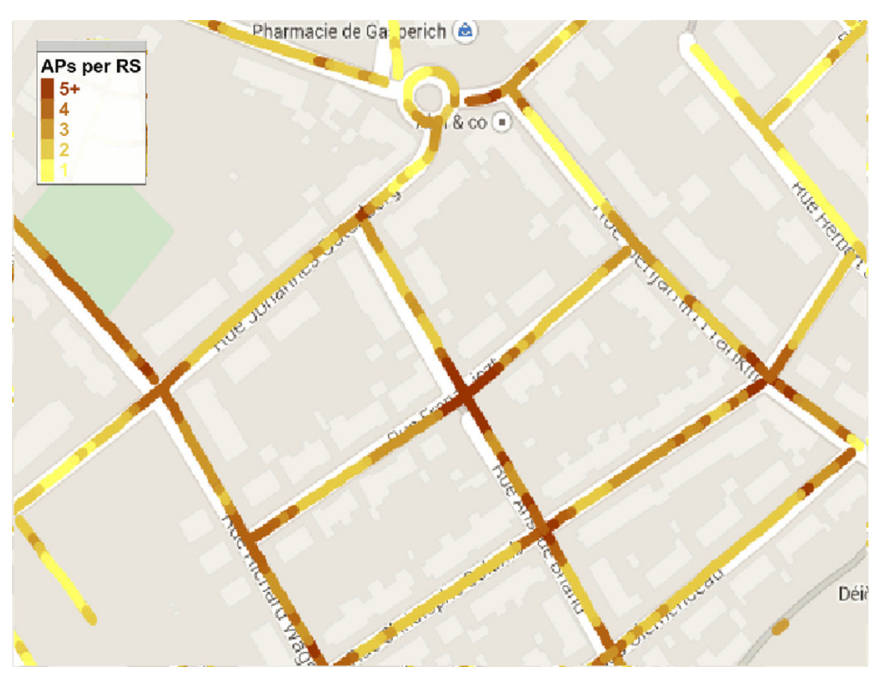

Fig. 2. Example of AP density heatmap based on the CDB.

RPs is lower than $10^{\circ}$. Otherwise, the candidate RP implies that a turn has been made. This threshold issues from the observation that intersections always comprise only one adjacent RP with an azimuth difference lower than $10^{\circ}$ which is not the case if one considers higher thresholds. The next direction is found by comparing the current trajectory of the vehicle with the characteristics the candidate RPs (i.e., the RPs starting at the next intersection). The current trajectory is defined by the two most recent vehicle locations $l_{t}$ and $l_{t-1}$, and the bearing of the vehicle $\beta$. The next direction detection starts when the vehicle enters the direction detection area (starting $15 \mathrm{~m}$ before the end of current RP). During this phase, the DDM computes the angular displacement $\gamma$ such that $\gamma=|\beta-\alpha|$. The DDM concludes the vehicle has entered an RP when $\gamma$ is lower than $20^{\circ}$ and the distance between vehicle and the RP entry point is decreasing (Eq. (1)). This threshold has been empirically obtained during a preliminary study. It results from a tradeoff between the risk of wrong detection and the objective of early detection.

$$
\left\{\begin{array}{r}
\gamma<20^{\circ} \quad(a) \\
d\left(l_{t}, D\right)<d\left(l_{t-1}, D\right) \quad(b)
\end{array}\right.
$$

If the DDM has not detected any turning $10 \mathrm{~m}$ after the end of the current RP, it considers that the vehicle is goes straight on. On one hand, this rule negatively impacts the performance of the DDM in terms of detection anticipation but on the other, it allows the DDM to detect a greater number of turning events. In addition, the early straight on event detection is considered less critical than the turning event detection due to the fact that the MS is more likely to stay longer in the line of sight of the current AP when the vehicle continues straight on.

Note that when the vehicle turns, its bearing often matches the azimuth of the next RP when the vehicle is about to enter to it. Therefore, in order to increase the anticipation of the vehicle's turning, we augment the DDM with a Fuzzy-based Turning Detector (FTD).

The FTD uses a fuzzy logic system to evaluate the probability that the vehicle is initiating a turn. The FTD relies on a simple model aiming at characterizing a turn based on the angular displacement $\gamma$. In our model, $\gamma$ is characterized by an evolution in two phases (phase I: initialization; phase II: stabilization) as depicted in Fig. 3. At start both $\gamma$ and the angular velocity $\gamma^{\prime}$ are zero, i.e. the vehicle is in the same direction than the current RP. In phase $I, \gamma^{\prime}$ increases from zero until reaching a maximum, the turning phase is initiated. In phase II, the angular velocity de-

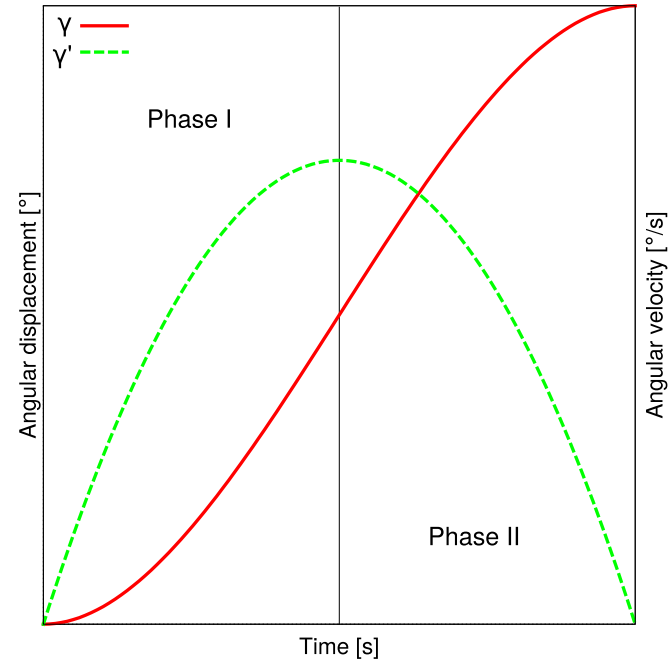

Fig. 3. Model of angular displacement during a turn.

creases until there is no more angular displacement. Entering in phase II indicates the initiation of trajectory stabilization.

The input variables of the fuzzy system are the vehicle speed, the acceleration (computed as the speed first derivative), $\gamma$ and the angular velocity $\gamma^{\prime}$ as the derivate of $\gamma$. In all cases, we consider trapezoidal membership functions. The output variable, the turning metric, is a value in the interval $[-180 ; 180]$ that is interpreted as the most likely angle of the vehicle's trajectory. We define seven linguistic variables for the turning metric: one for the non-turning case and three (low, medium and high) for each turning direction (i.e., negative values imply turning left while positive values imply turning right). The FTD considers a set of rules that can be summarized as follows. (i) There is no turn (i.e., turning metric equals zero) if $\gamma$ is low or the vehicle speed is high. (ii) If the vehicle speed is low and there is negative acceleration, the turning likelihood is high. (iii) If the angular rate is non-zero and $\gamma$ is increasing, the turning likelihood is high (phase I in Fig. 3). (iv) If the angular rate is null or $\gamma$ is decreasing, the turning likelihood decreases (phase II in Fig. 3). And finally, (v) If the vehicle is driving off (i.e. low speed and high acceleration), the turning likelihood increases. Note also that the angular rate is used here as a filter since it indicates whether or not the turning movement keeps going (see the fourth and fifth rules). In order to select the candidate RPs we evaluate the relative angle between the azimuth of the current AP and each candidate. We define seven groups: one for the RP that implies the vehicle is continuing straight on (relative angle below $10^{\circ}$ ), and three groups (for turning right and left) each for RPs that are linked to a low, medium or high turn for relative angles between $10^{\circ}$ and $45^{\circ}, 45^{\circ}$ and $110^{\circ}$ and higher than $110^{\circ}$ respectively. Note that the case where two candidate RPs are classified in the same group is not frequent and considered out of the scope of this study. In contrast to the previous approach, the FTD is able to anticipate the vehicle's direction. However, the FTD can only be used to detect turning; thus, the DDM combines these two approaches and considers the one providing the earliest detection.

\subsection{AP selection module}

The ASM intends to find the AP providing the best network connectivity based on the direction taken by the vehicle. As aforementioned, the MS does not know the complete route of the vehicle in advance. This mean that AP selection must be triggered every time the vehicle enters a new RP. In a first phase, the MS evaluates possible handovers to candidate APs located at the roadside of 


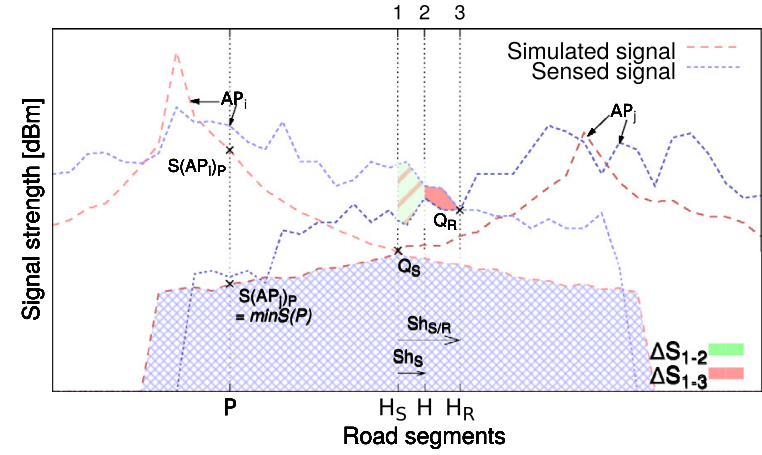

(a) Case 1

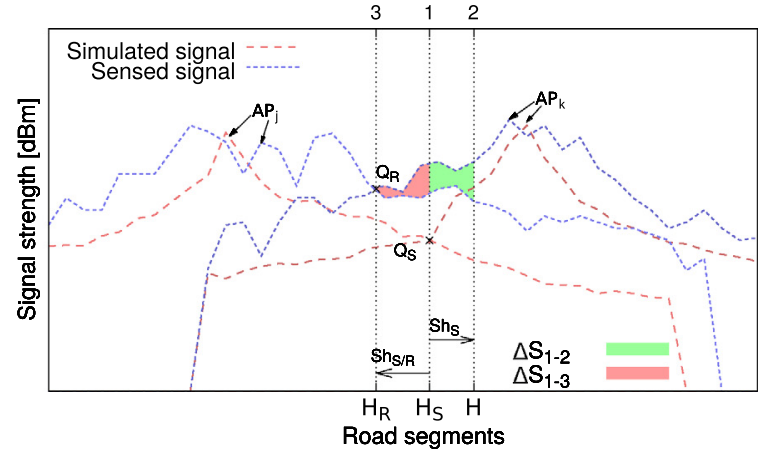

(b) Case 2

Fig. 4. Sample of simulated and sensed RSS.

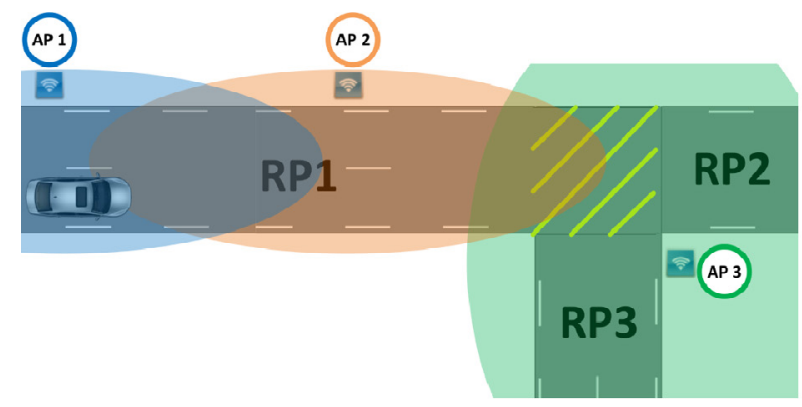

(a) Case 1

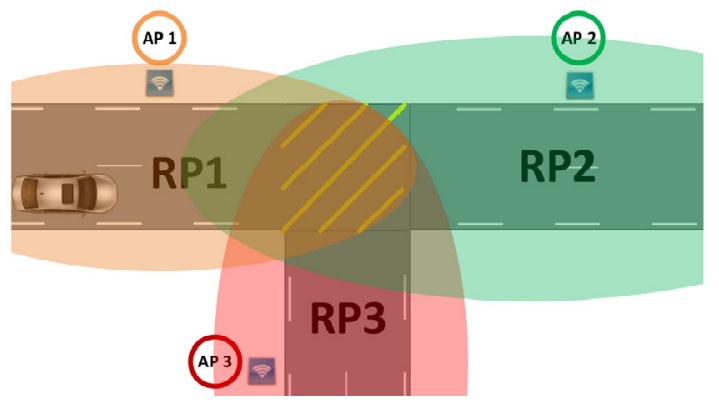

(c) Case 2B

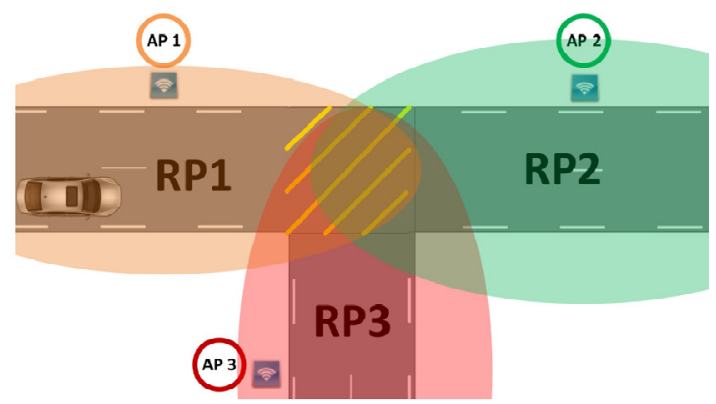

(b) Case $2 \mathrm{~A}$

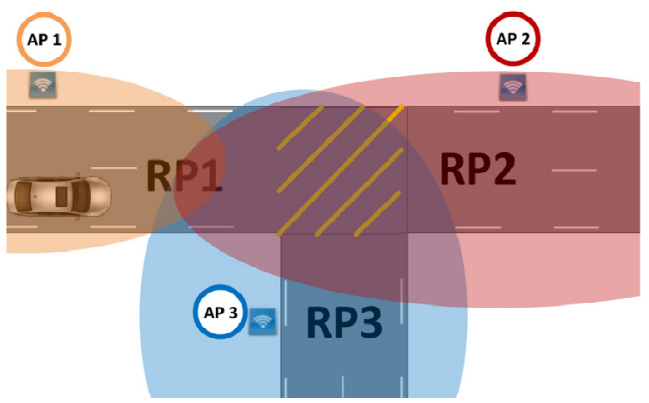

(d) Case 3

Fig. 5. The different cases handled by the ASM.

the current RP. It, then, evaluates handovers to APs located at the roadside of next RPs and makes an anticipated handover decision if possible. The selected APs are then stored in the candidate AP list that is used by the connection module (cf. Fig. 1).

\subsubsection{Handover evaluation and localization}

For each candidate AP, the ASM builds a handover quality metric that is used in the AP selection. In addition, the ASM computes the handover location as the location where the RSSs of both the current and the candidate AP are simultaneously maximized. Fig. 4(a) shows a sample of the simulated and real RSSs of two APs called respectively $A P_{i}$ (current AP) and $A P_{j}$ (candidate AP).

Note that the CDB only provides the modeled RSS to the ASM. The sensed RSS is only used to evaluate ASM performance. The handover computation consists of three steps. First, the ASM defines the overlapping area $\left(O v_{S}\right)$, i.e., the set of RPs where the MS is able to receive a signal from both $A P_{i}$ and $A P_{j}$.

Second, the ASM selects for each RS the lowest RSS. Let $\operatorname{minS}(P)$ in Eq. (2) be the lowest signal function that is evaluated over an RS $P \in O v_{S}$.

$\min S(P)=\min \left(S\left(A P_{i}\right)_{P}, S\left(A P_{j}\right)_{P}\right)$
In Fig. 4(a), for the example of the RS $P, \min S(P)$ is $S\left(A P_{j}\right)_{P}$. The set of all the elements of the lowest signal function is shown by the curve delimiting the pattern-filled area.

Third, the ASM selects the handover location as the RS maximizing Eq. (2).

In Fig. 4(a) we can see the handover signal quality $Q_{S}$ as the maximum of the curve delimiting the pattern-filled area and the handover location $H_{S}$, the RS corresponding to $H S_{S}$.

\subsubsection{AP selection process}

The AP selection is triggered every time a new RP is detected by the DDM. The ASM automatically selects the APs located in this new RP and explores potential handovers toward an AP located in the next possible RPs. The ASM classifies the network deployment after the selected RP into three groups that cover all the possible scenarios. These scenarios are depicted in Fig. 5. First, the ASM looks for a single common AP among the next RPs (Fig. 5(a)). If such an AP is found, this AP is selected, the handover location is computed and the AP selection is resumed the next time the DDM detects a new RP. If not, the ASM analyzes the RSS of the current AP. If this RSS is high enough up to the end of the RP, AP selection ends (Fig. 5(b) and Fig. 5(c)). If not, the ASM forces the selection of 
another AP located in the next RPs (e.g., AP2 or AP3 in Fig. 5(d)). Note that when an AP is selected, the ASM computes the handover location $\left(H_{S}\right)$ as described before and provides the $\mathrm{CM}$ with the 3-tuple $\left\{A P_{\text {from }}, A P_{\text {to }}, H_{S}\right\}$ where $A P_{\text {from }}$ is the currently selected AP and $A P_{\text {to }}$ is the next selected AP.

Note that since the AP selection process relies on offline information, the ASM cannot guarantee that the candidate AP is available and can provide enough bandwidth to the MS. In case the candidate AP is unavailable, the MS should be able to attempt a connection to a different candidate AP without extra delay. Therefore, for each AP, the ASM should provide a long-term evaluation of the potential candidate APs in the current RP and after. That is, the ASM should consider all the possible transitions between the current AP and next candidate APs (inside and outside current RP) and classify them. In case the candidate AP selected by the regular AP selection is unavailable, the MS directly choose the next AP in the classified candidate APs list. Some classification methods are discussed later on this paper.

We can identify two situations where the MS is not associated with the best AP. The first is due to an incorrect estimate of the RSS, leading to situations where the handover location based on the simulated RSS $\left(H_{S}\right)$ does not correspond with the optimal handover location in the reality $\left(H_{R}\right)$. The spatial shift between those two handover locations is $S h_{S / R}$ (see Figs. 4(a) and 4(b)). $S h_{S / R}$ is considered positive if $H_{R}$ is located after $H_{S}$ and negative otherwise. For a given period, the RSS of the current AP is lower than the RSS of the best AP. This RSS difference is called $\Delta S_{1-3}$. The second suboptimal AP selection case is due to the short-term prediction of vehicle's direction. As the DDM often detects the next $\mathrm{RP}$ at the end of current RP the ASM delays the AP selection when the current AP can provide good signal until the end of current RP. This sometimes leads to suboptimal AP selection. In case 3 of the illustration (Fig. 5(c)), AP2 and AP3 provide higher RSS at the end of the current RP. In this case the AP selection is suboptimal since the ASM does not select the best available AP. This situation is shown in Figs. 4(a) and 4(b)), where the actual handover location $H$ does not correspond to best handover location $H_{S}$. The spatial shift between the two is denoted as $\mathrm{Sh}_{S}$ and the RSS difference, as $\Delta S_{1-2} . S h_{S}$ is considered always positive since $H$ could never be ahead of $H_{S}$.

\subsection{Connection module}

The $\mathrm{CM}$ is in charge of triggering a new association with the selected APs at the handover location given by the ASM. This module only takes as input the selected AP list and the current vehicle location without performing any AP scanning. Fig. 1 describes the connection procedure followed by the $\mathrm{CM}$. First, the $\mathrm{CM}$ parses the selected AP list by comparing the selected AP handover location with the current location of the vehicle. If the handover location of a selected AP is reached or outdistanced, the CM starts the procedure for connection to this AP. The connection procedure consists of two phases. First, since the CM does not perform any scan, it does not know if the selected AP is actually available or not. Indeed, the fact that the selected AP is listed in the CDB does not guarantee that this AP is actually available. As a result, in order to check the availability of the AP, the CM sends a Probe Request to this AP. If the CM does not receive any Probe Response after three attempts, the selected AP is considered unavailable. The ASM, then, performs a new AP selection ignoring this unavailable AP. In contrast, if a Probe Response is received from the selected AP, the CM initiates the association process. This process consists of the standard IEEE 802.11 authentication and association, i.e., the exchange of four messages (authentication request/response and association request/response) with the selected AP. If this phase fails, the $\mathrm{CM}$ retries a connection to the selected AP.

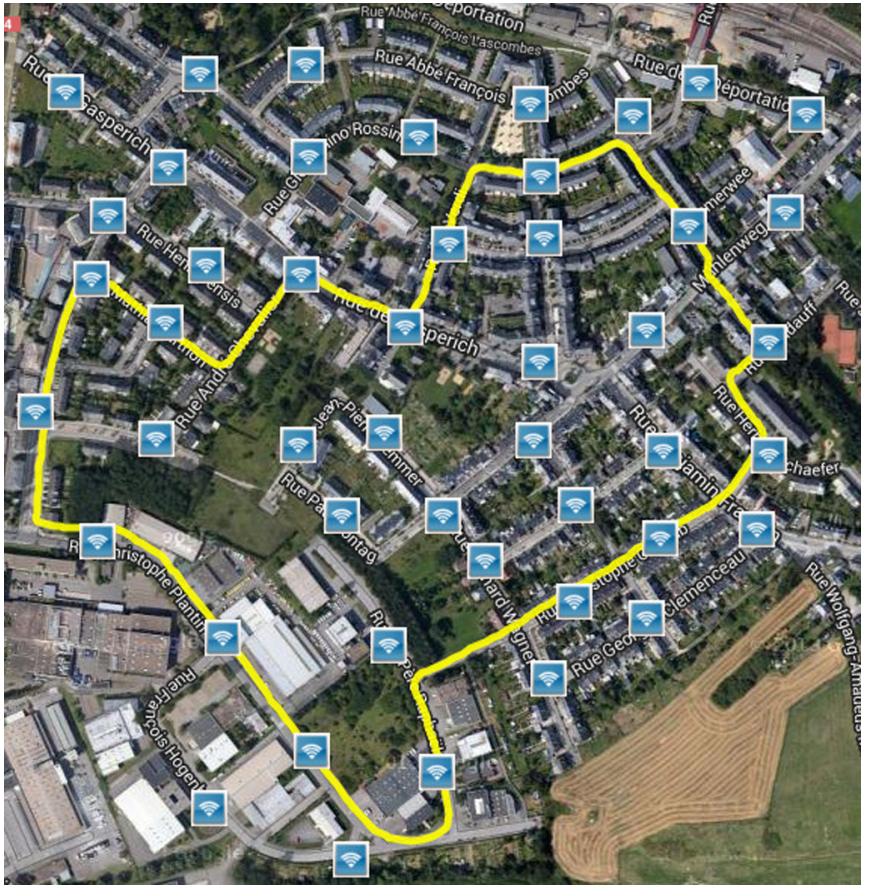

Fig. 6. Map of the experimental path.

\section{Evaluation}

\subsection{Testbed setup}

In order to evaluate the performance of COPER, we performed a set of experiments using the HotCity network, a metropolitan IEEE 802.11 network deployed over a large part of the city of Luxembourg. HotCity provides Internet access to mobile users in multiple areas of the city. It is composed of around 500 Cisco Aironet APs for outdoor usage, embedding an IEEE $802.11 \mathrm{~b} / \mathrm{g}$ radio for client wireless access and an IEEE 802.11a radio for mesh interconnection between APs. HotCity is an open WiFi that uses Hyper Text Transfer Protocol (HTTP) authentication for registered users wanting to access the network. A detailed connection performance study of HotCity appears in [28]. Note that the scenario considered in building the CDB includes a subset of 392 APs from the HotCity network, for which information is available online. ${ }^{2}$ Fig. 6 shows the experimental route used to evaluate coPER. This route is located in Gasperich, a residential neighborhood of Luxembourg city. This route is $2.6 \mathrm{~km}$ long and comprises 18 roadside APs along the path. The road signals include one traffic light and multiple stop signs and intersections. However, since the speed limit is low (i.e., $30 \mathrm{~km} / \mathrm{h}$ ) on almost the complete experimental route, the duration of each individual experiment remains almost constant. The average duration of a single experiment is $353 \mathrm{~s}$ with a standard deviation of $14 \mathrm{~s}$. Fig. 7 highlights the number of APs and the maximum RSS detected in all the RSs of the experimental path. The distribution of the number of APs detected shows that there is no disconnected area on the experimental route. Also, the highest RSS sensed in each RS of the experimental route is always greater than $-80 \mathrm{dBm}$. Note that, as shown in Fig. 8 , an RSS lower than $-80 \mathrm{dBm}$ significantly degrades the QoS. These results are consistent with [29]. As a result, in the best case the MS is associated with a HotCity AP and has Internet connectivity all along the experimental route. The MS used in these experiments was a

2 www.topographie.lu. 

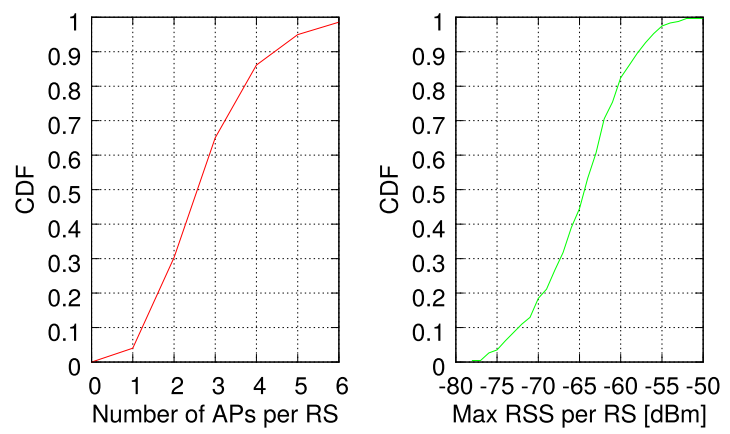

Fig. 7. CDF of the number of AP per RS and mean data rate per RSS.

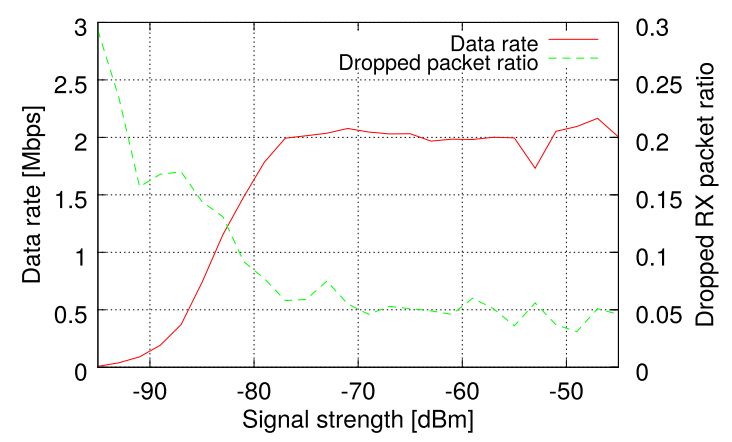

Fig. 8. Relationship between RSS and data rate and dropped packets.

Nexcom VTC 6200-NI-DK ${ }^{3}$ Linux-based embedded platform implementing a Kernel v3.11. The experiments were performed with the bulk IEEE $802.11 \mathrm{~b} / \mathrm{g} / \mathrm{n}$ mini PCI wireless card using Ralink RT3092 chipset and a Pulse multiband antenna ${ }^{4}$ providing $6 \mathrm{dBi}$ gain in the $2.4 \mathrm{GHz}$ band.

In order to provide meaningful results, the performance of COPER is compared to other solutions: (i) network manager ${ }^{5}$ (netMan), (ii) a modified version of WPA-supplicant having an RSS threshold of $-75 \mathrm{dBm}$ and (iii) MROAD [23]. We consider in this comparative study netMan and WPA-supplicant in order to evaluate how legacy scanning-based approaches perform compared to COPER and MROAD that are specifically designed for vehicular communications. The comparison between COPER and MROAD intends to investigate the eventual impact of the direction detection process on MS communication. The experiments are performed as follows. Before an experiment starts, the MS obtains an IP address through DHCP and keeps it for the whole experiment, since the HotCity network supports handovers at layer 2. When the vehicle is at the departure point, the MS initializes a 2.5 Mbps downlink User Datagram Protocol (UDP) flow from a remote server in our laboratory. During the experiment, we capture the received packets using tcpdump and record a log at the MS, including the Linux kernel $\log$, the GPS location of the vehicle and the network interface statistics (e.g., RSS of current AP, amount of data received and transmitted, packet loss). The experiments are repeated five times for each considered solution in order to obtain representative averaged values.

\subsection{Evaluation of the direction detection}

First, we investigate the DDM performance. The experimental route for this experiment is $6.5 \mathrm{~km}$ long and comprises 62 inter-

\footnotetext{
3 http://www.nexcom.com/Products/mobile-computing-solutions/in-vehicle-pc/invehicle-pc/transportation-computer-vtc-6200.

4 http://productfinder.pulseeng.com/productSearch/gpsdm700.

5 https://wiki.gnome.org/Projects/NetworkManager.
}

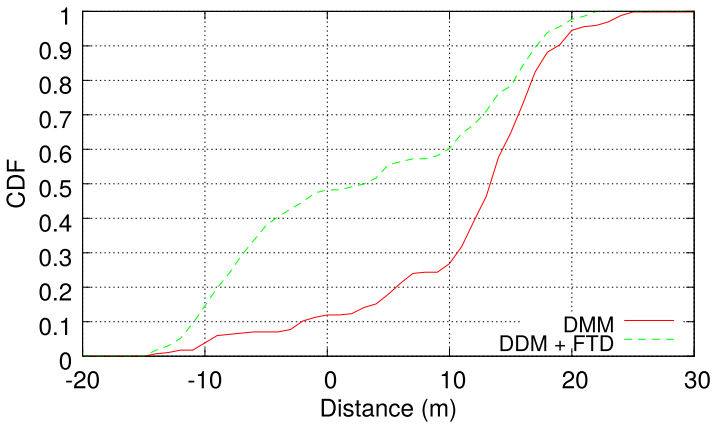

Fig. 9. CDF of anticipation distance.

Table 1

Turn detection performance of DDM and DDM with FTD.

\begin{tabular}{lll}
\hline & Median detection & Detection $<10 \mathrm{~m}$ \\
\hline DMM & $12 \mathrm{~m}$ & $45 \%$ \\
DMM + FTD & $-7 \mathrm{~m}$ & $92 \%$ \\
\hline
\end{tabular}

sections where the vehicle performs 43 turns. This scenario includes multiple types of intersection, implying different mobility patterns for the vehicle (i.e., different speeds and trajectories). We used a $4 \mathrm{~Hz}$ GPS receiver in order to have a frequent update of the vehicle's trajectory and decrease detection latency. The experiment was repeated multiple times in order to obtain a representative overview of the detection performance of the DDM. As mentioned in Section 3.2, the most critical part of the direction detection is turn detection: when the vehicle turns at an intersection, the MS has more chances to leave the line of sight of the current AP, thus facing a significant decrease of current AP RSS. Table 1 highlights the performance in terms of turning detection for the standalone DDM and the DDM combined with the FTD. We use the distance between the detection location and the end of the current RP as performance metric. This distance is negative when the detection occurs before the vehicle reaches the end of the current RP (anticipated detection) and positive otherwise. We also evaluated the false negative rate, which corresponds to the case where the DDM does not detect that the vehicle is turning or selects an incorrect $\mathrm{RP}$, and the false positive case corresponds to the case where the system detects a turn but the vehicle is not turning. We obtained seven false positives and seven false negatives against 129 successful detections (95\%) for both standalone DDM and DDM augmented with FTD. This suggests that the addition of the FTD does not have an impact on the detection success of the DDM. However, FTD provides an enhancement to median detection anticipation of $19 \mathrm{~m}$. In addition, $92 \%$ of the detections occur below $10 \mathrm{~m}$ versus $45 \%$ for the standalone DDM. If we focus on overall direction detection (straight RPs and turnings), as depicted in Fig. 9, we observe a median detection anticipation of $10 \mathrm{~m}$ when DDM is augmented through FTD ( $3 \mathrm{~m}$ vs. $13.2 \mathrm{~m}$ ). This anticipation performance has a significant impact on the quality of AP selection, in particular when the vehicle is about to turn. An early detection of the vehicle's direction allows the ASM to detect when the RSS of the current AP could decrease in the short-term and to anticipate the selection of the next AP such that the handover is triggered before the current AP's RSS falls dramatically.

\subsection{Evaluation of AP selection}

In this section, we focus on the study of the COPER AP selection process. As previously mentioned, AP selection is suboptimal when the handover decision is delayed until the next predicted 

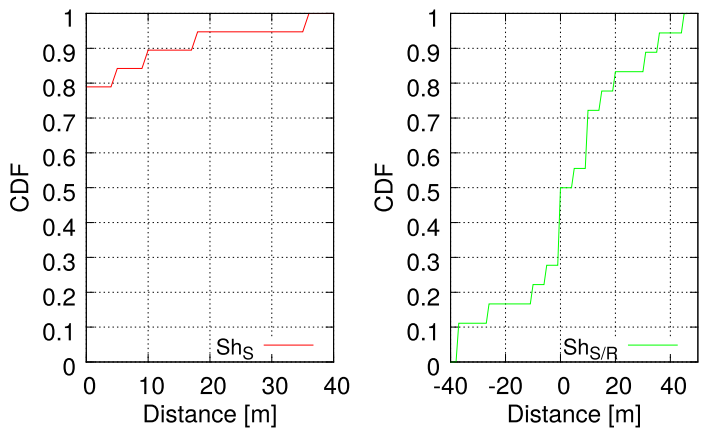

Fig. 10. $\mathrm{CDF}$ of $S h_{S}$ and $S h_{S / R}$.

Table 2

Distribution of $\Delta S$ when $\Delta S>0$.

\begin{tabular}{llll}
\hline Overall \% of RS & $\begin{array}{l}\text { Median } \\
\text { value }(\mathrm{dB})\end{array}$ & $\begin{array}{l}\text { Relative \% of RS } \\
\text { where } \Delta S<5 \mathrm{~dB}\end{array}$ & $\begin{array}{l}\text { Relative \% of RS } \\
R S S<-80 \mathrm{dBm}\end{array}$ \\
\hline 19.3 & 2.6 & 70.1 & 2.8 \\
\hline
\end{tabular}

direction is taken or when the handover is misplaced due to an incorrect RSS estimation. The handover spatial shifts caused by these two issues are analyzed in Fig. 10, which shows that the handover location shifts due to the lack of information about the vehicle's direction are much shorter than those caused by the erroneous estimation of the RSS. We can see that $S h_{S}$ is equal to zero in almost $79 \%$ of the cases against $21 \%$ for $S h_{S / R}$. Moreover, $S h_{S}$ is, in general, below $20 \mathrm{~m}$ whereas the $S h_{S / R}$ is rather uniformly distributed in the range $[-40 \mathrm{~m} ; 40 \mathrm{~m}]$. We can conclude that the handover is misplaced mainly due to the difference between the simulated RSS (stored in the CDB) and actual RSS. The distribution of the RSS difference between the current and best AP (see Table 2) reveals that the RSS of the current AP is the best available in more than $80 \%$ of the RSs of the run. The misplacement of handover compared to the ideal location has a negligible impact on the RSS. Indeed, the median RSS difference relative to the best AP is only $2.8 \mathrm{~dB}$ and in $70.1 \%$ of the cases this value is under $5 \mathrm{~dB}$. In addition, cases where suboptimal AP selection implies a critically low RSS (i.e., below $-80 \mathrm{dBm}$ ) are not frequently observed (i.e., in only $2.8 \%$ of the RS we observed $\Delta S>0$ ). As a result, we conclude that CoRNER modeled RSSs are realistic enough to be used for AP selection.

\subsection{Connection performance of the MS}

In this section we analyze several metrics related to MS connectivity during the experiments: the data rate of the downlink UDP flow at the MS, the MS disconnection time and the RSS of the current AP.

\subsubsection{Network performance comparison}

Fig. 11(a) shows the distribution of the mean data rate of the downlink UDP flow. From this figure we can observe that the total time where the MS does not receive any data from the network is much longer for both netMan and WPA-supplicant based handover (around 20\%) than for MROAD and COPER (less than 1\%).

Table 3 shows that MROAD and COPER have both high associated time (above 99\%) and high packet delivery ratio (above 95\%). In the same way, netMan and WPA-supplicant have approximatively the same packet delivery ratio (around $80 \%$ ). Note that the packet delivery ratio is an estimate since in our experiments, the MS a flow of $2.5 \mathrm{Mbps}$ which is higher than the capacity of the network.

Fig. 11(b) shows the distribution of the disconnected time for all the considered approaches. The median value for the disconnected time is around $1200 \mathrm{~ms}$ (maximum $5385 \mathrm{~ms}$ ) for WPAsupplicant and $1300 \mathrm{~ms}$ (maximum $7684 \mathrm{~ms}$ ) for netMan versus around $100 \mathrm{~ms}$ (maximum $2277 \mathrm{~ms}$ and $1325 \mathrm{~ms}$ ) for MROAD and COPER respectively. This is explained by the fact that WPAsupplicant and netMan trigger AP scanning at each handover and could partially explain why the MS receives no data during around $20 \%$ of the tune using these solutions (see Fig. 11(a)). We conclude that avoiding the scan significantly reduces disconnection periods.

Further, the study of the distribution of the RSS (Fig. 11(c)) shows again a large difference between the two sets of solutions. We observed an RSS lower than $-80 \mathrm{dBm}$ in $21 \%$ and $33 \%$ of the cases for WPA-supplicant and netMan respectively, while a signal level this low was observed in only $3.3 \%$ and $2.8 \%$ of the case for MROAD and COPER respectively. Also, the median observed RSS is around $-64 \mathrm{dBm}$ for MROAD and COPER versus $-67 \mathrm{dBm}$ for WPAsupplicant and $-71 \mathrm{dBm}$ for netMan. Again, the distribution of the RSS of MROAD and COPER are similar and indeed close to the distribution of the maximum RSS sensed over the experimental path (Fig. 7). Fig. 8 shows that when the RSS drops below $-80 \mathrm{dBm}$, the data rate decreases significantly.

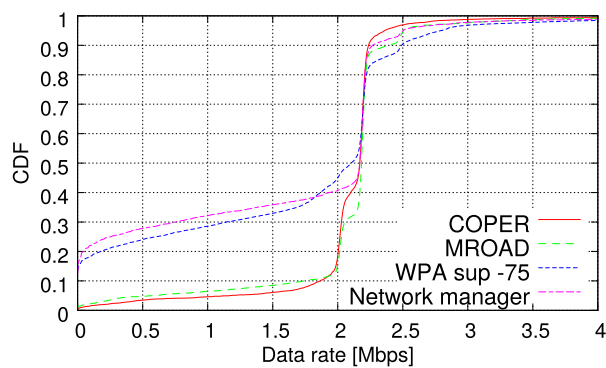

(a) CDF of the data rate

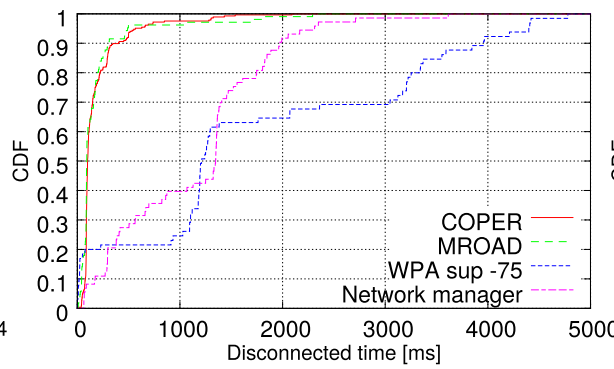

(b) CDF of the disconnected time

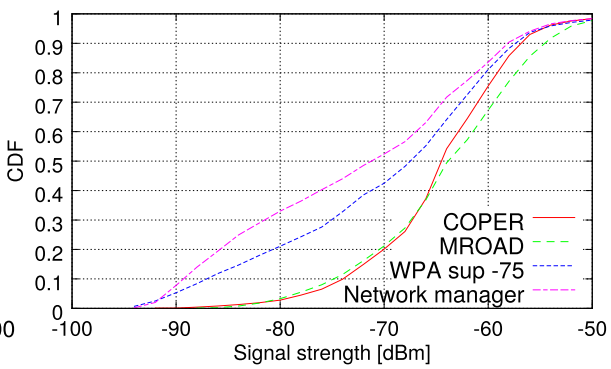

(c) CDF of the sensed RSS

Fig. 11. Comparative results.

Table 3

Comparative performance of different handover solutions with confidence interval ${ }^{\mathrm{a}}$.

\begin{tabular}{|c|c|c|c|c|c|}
\hline & $\begin{array}{l}\text { Estimated packet } \\
\text { delivery ratio }\end{array}$ & Associated time & $\begin{array}{l}\text { Number of AP } \\
\text { association per run }\end{array}$ & $\begin{array}{l}\text { Distinct AP associated } \\
\text { (outside roadside) }\end{array}$ & $\begin{array}{l}\text { Mean connected } \\
\text { time }\end{array}$ \\
\hline NetMan & $0.79 \pm 0.05$ & $88.2 \pm 0.68$ & $15.4 \pm 1.17$ & $15(3)$ & $23.79 \pm 13.25$ \\
\hline WPA sup -75 & $0.80 \pm 0.02$ & $78.8 \pm 0.47$ & $39.7 \pm 3.12$ & $25(6)$ & $7.15 \pm 0.87$ \\
\hline MROAD & $0.97 \pm 0.03$ & $99.06 \pm 0.17$ & $19 \pm 0.00$ & $18(0)$ & $15.25 \pm 1.43$ \\
\hline COPER & $0.95 \pm 0.02$ & $99.08 \pm 0.14$ & $19 \pm 0.00$ & $18(0)$ & $17.17 \pm 1.33$ \\
\hline
\end{tabular}

\footnotetext{
a Corresponding to a confidence level of $95 \%$ considering that the population have a normal distribution.
} 
In contrast, the distributions for netMan and WPA-supplicant differ significantly. NetMan is more affected by low RSS than WPAsupplicant but this seems to have no impact on the data rate nor on the packet delivery ratio. We observe that the RSS distribution for values greater than $-65 \mathrm{dBm}$ for COPER and MROAD are not equal. This could be because AP selection for COPER is sometimes delayed compared to MROAD (cf. Section 4.3). Nevertheless, this has no significant impact on the data rate because, due to ISP bandwidth capping, all the RSS above $-77 \mathrm{dBm}$ provide equivalent data rates (cf. Fig. 8).

Now we consider the associated time, the number of successful associations, the mean associated time shown in Table 3. The associated time starts when the MS associates with an AP and ends at the beginning of the scan that precedes the next handover. These results show that, using MROAD and COPER, the MS is almost always associated with an AP (99\% of the tune) while this is the case for only $88.2 \%$ and $78.8 \%$ of the time with netMan and WPA-supplicant respectively. We observe a significant difference of the mean connected time with these two solutions. The MS remains connected with an AP on average 23.79 s for NetMan and $7.15 \mathrm{~s}$ for WPA-supplicant versus $15.25 \mathrm{~s}$ and $17.17 \mathrm{~s}$ for MROAD and COPER respectively. In the case of WPA-supplicant, the higher re-connection frequency leads to shorter connection duration, which increases the instability of the MS connection contrary to netMan which is even more durably connected than MROAD and COPER. We observed in Fig. 11(b) that WPA-supplicant causes long disconnection due to scanning, thus, this solution is more impacted by disconnections than netMan because it triggers handovers much more often. Here we realize that both netMan and WPA-supplicant are affected by two different factors, poor link quality during long periods for the first and frequent disconnections for the second, that seems to have a similar impact as both have the same data rate distribution and packet delivery ratio. These results also show that MROAD and COPER allows long and stable associations (the sequence of selected APs remains the same over the runs). We note instability of the AP selection being based only on instantaneous scanning results, from which both netMan and WPA-supplicant select between three and eight APs outside the route of the vehicle, which are unable to provide a sustainable RSS to the MS. Also, the APs located at the roadside are not always selected in their order of appearance. In contrast, MROAD and COPER select only the 18 APs providing the best signal all along the path.

Note that COPER outperforms MROAD in terms of disconnection time. Three factors can be cited to explain this slight difference of performance. The first is the handover spatial shifts caused by the lack of information about the vehicle's direction, highlighted in previous section. This may also be due to the experimental conditions not being exactly the same when evaluating MROAD and COPER and because the performance of a given solution can vary slightly from one run to the other. Nevertheless, we can conclude that by using COPER as a predictive handover mechanism, we can provide similar performance to MROAD but without requiring any prior knowledge of the complete path of the vehicle, which greatly increases flexibility.

\subsubsection{Impact of the AP selection and handover process}

Finally, we study the impact of the AP transition process on MS connectivity. We distinguish between the AP selection phase, which involves the offline computation to find the best AP, and the handover execution, where the MS probes the selected AP and associates to it. Fig. 12 illustrates the duration of the these two phases.

This offline computation implies that the AP selection is performed by the ASM and the candidate AP list parsing by the CM before attempting a handover. The median duration of the ASM AP selection and the candidate AP list parsing are $340 \mathrm{~ms}$ and $27 \mathrm{~ms}$

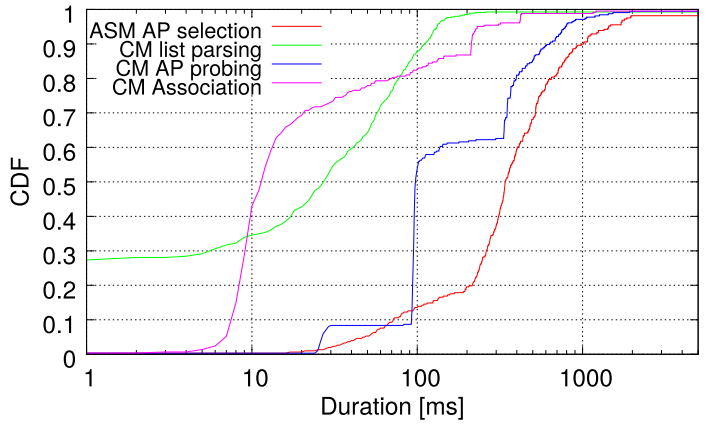

Fig. 12. CDF of the handover phases duration.

respectively. This is a long process compared to the handover execution, that has a median of $12 \mathrm{~ms}$ for AP probing and $100 \mathrm{~ms}$ for authentication and association. However, AP selection in COPER is performed offline, preventing a disconnection between the MS and the current AP. In fact, the duration of the AP selection phase reflects the reaction time of COPER when a new RP is discovered, thus, in the worst case, AP selection only slightly delays handover.

During the probing phase in COPER, the MS sends a broadcast Probe Request on the channel where the selected AP is operating and waits for Probe Responses from all APs in this channel for a fixed time while in the standard handover, the MS repeats the same process on all channels. This results in a much shorter median duration for COPER (98 $\mathrm{ms}$ ) than for the standard handover used by netMan (1143 ms). Note that the AP probing duration distributions shows three modes that correspond to (i) the case where the MS retrieves the selected AP in recently-stored scan results ( $\sim 20 \mathrm{~ms}$ ), (ii) the MS received a Probe Response from the selected AP after the first attempt ( $\sim 100 \mathrm{~ms})$ and (iii) the MS needs several attempts to probe the selected AP (AP probing duration greater than $200 \mathrm{~ms}$ ).

The handover phase of COPER uses probing and association methods available in the existing Linux kernel module, which could be optimized. For instance, the probing could be improved by specifically targeting the SSID of the selected AP, sending multiple probes within a short time interval or timing out sooner while waiting for Probe Responses.

\section{Discussion}

The approach proposed in this work has been evaluated in a controlled environment: a city-wide 802.11 hotspot network characterized by a uniform AP density and layer 2 handover support. The advantages of such an environment are the ease of gathering all the information needed to model the network, and the fact that the network topology does not change frequently, meaning that the CDB remains reliable over time. Note that COPER could operate in city-wide $802.11 \mathrm{p}$ deployments that consist of a fixed set of RSUs since the characteristics of such networks are similar to the one we evaluated in terms of coverage range as shown in [30]. This study, intended to evaluate IEEE 802.11p RSUs in many mobility patterns, shows that they can provide a reliable connectivity (packet delivery ratio $>0.7$ ) to a mobile vehicle between $100 \mathrm{~m}$ and more than $750 \mathrm{~m}$. However, the fact that the RSU may be able to provide some contextual information to the MS on a dedicated channel (e.g., channel load, number of MSs connected to the RSU, delay, jitter, packet loss) opens the way to dynamically feed the CDB with relevant information for handover decision making. This would allow the MS to prioritize some candidate APs on the fly, according to the contextual information, rather than only basing the AP selection on the current location of the vehicle. In this case, the selection of the best AP could be based on a Multi Attribute Decision Making technique [31]. 
Further, the ever-growing number of residential and community networks offers widespread coverage in urban and suburban environments. At the same time, cellular networks have been deployed over a great part of the populated regions of the world. COPER could be extended to all these heterogeneous networks. However, a high number of APs and frequent changes in network infrastructure deployments leads to open questions concerning information collection on these networks and the dynamic evolution of the CDB to keep it up-to-date.

As a result, solutions are needed to enable the dynamic collection and incorporation of available network and mobility information and in the database. A collaborative approach based on the actual experience of users is one possible strategy. Here, the MS periodically collects contextual information on the networks (e.g., RSS, packet loss, data rate) through a Mobile CrowdSourcing (MCS) application [32]. The collected information, stored and analyzed in the cloud, could be used to detect out-of-order APs, improve the model used by the simulation framework, and to build a temporal profile of each AP that could be considered in the AP selection.

In order to improve COPER reliability, the AP selection should focus on the long-term evolution of the network environment. To this aim, an AP classification mechanism has been previously described that intends to allow the MS selecting with no delay a new candidate AP in case the selected one is not available. Considering only the information stored in the $\mathrm{CDB}$, the classification may be performed based on the handover quality parameter. In addition, it could be improved by integrating a long-term mobility prediction based on the analysis of the historical traces collected, for instance, by the mean of the MCS application mentioned before. This approach would allow the MS to make a pre-selection of the AP that will be probably visited.

\section{Conclusion}

In order to provide an ubiquitous network connection through 802.11 networks to mobile vehicles, the standard IEEE 802.11 handover procedure needs to be amended with the aim of avoiding long disconnections and potentially inconsistent AP selections. Although it has been proven that the disconnected time resulting from scanning can be significantly reduced, the lack of information on the short-term evolution of the APs' RSSs suggests that other of sources of information should be considered in order to provide a more accurate view of network evolution over the short term.

In this work we have presented COPER, a predictive handover mechanism that replaces the scanning procedure by using prior knowledge of the road topology and the modeled APs RSS. Based on this information and knowledge of the vehicle's predicted direction, COPER allows the MS to select best next APs in the light of the short-term evolution of their RSSs. This approach shortens the handover to a single channel probing and the authentication/association phase, which significantly reduces the handover latency. Our field experiments conducted on a city-wide 802.11 network show that this handover optimization materially decreases the time when the MS is disconnected. The average data rate using COPER remains close to the maximum achievable data rate on nearly the whole duration of the experiments.

We have showed that COPER can significantly improve the network connectivity of a vehicle connected to large 802.11 networks, which are characterized by a uniform AP density comparable to future 802.11 p deployments. However further investigation is needed to evaluate such a solution on heterogeneous 802.11 networks (e.g., residential and community 802.11 networks) that have different characteristics (e.g., high number of APs, shorter coverage, frequent topology changes). In addition, some research should be done into the best way to run such experiments on a larger scale.

\section{Acknowledgements}

The authors would like to thank the Luxembourg National Research Fund (FNR) for providing financial support through the CORE 2010 MOVE project (C10/IS/786097). We also want to thank Ismail Yildiz for his contribution.

\section{References}

[1] Sang-Hee Park, Hye-Soo Kim, Chun-Su Park, Jae-Won Kim, Sung-Jea Ko, Selective channel scanning for fast handoff in wireless LAN using neighbor graph, in: Personal Wireless Communications, Springer, 2004, pp. 194-203.

[2] Arunesh Mishra, Minho Shin, William Arbaugh, An empirical analysis of the IEEE 802.11 MAC layer handoff process, Comput. Commun. Rev. 33 (2) (2003) 93-102.

[3] German Castignani, Andrés Arcia, Nicolas Montavont, A study of the discovery process in 802.11 networks, Mob. Comput. Commun. Rev. 15 (1) (2011) 25-36.

[4] IEEE Standard for Information technology - local and metropolitan area networks - specific requirements - Part 11: Wireless LAN Medium Access Control (MAC) and Physical Layer (PHY) specifications amendment 6: wireless access in vehicular environments, in: IEEE Std 802.11p-2010, July 2010, pp. 1-51.

[5] Eva Gustafsson, Annika Jonsson, Always best connected, IEEE Wirel. Commun. 10 (1) (2003) 49-55.

[6] Julien Montavont, Nicolas Montavont, Thomas Noel, Enhanced schemes for L2 handover in IEEE 802.11 networks and their evaluations, in: IEEE 16th International Symposium on Personal, Indoor and Mobile Radio Communications, vol. 3, PIMRC 2005, IEEE, 2005, pp. 1429-1434.

[7] Ishwar Ramani, Stefan Savage, SyncScan: practical fast handoff for 802.11 infrastructure networks, in: 24th Annual Joint Conference of the IEEE Computer and Communications Societies, INFOCOM 2005, in: Proc. IEEE, vol. 1, IEEE, 2005, pp. 675-684.

[8] Kishore Ramachandran, Sampath Rangarajan, John C. Lin, Make-before-break MAC Layer Handoff, in: IEEE International Conference on 802.11 Wireless Networks Communications, ICC'06, vol. 10, IEEE, 2006, pp. 4818-4823.

[9] Vladimir Brik, Arunesh Mishra, Suman Banerjee, Eliminating handoff latencies in 802.11 WLANs using multiple radios: applications, experience, and evaluation, in: Proceedings of the 5th ACM SIGCOMM Conference on Internet Measurement, USENIX Association, 2005, p. 27.

[10] Maximilien Mouton, German Castignani, Raphaël Frank, Lara Codeca, Thomas Engel, On the evaluation of make-before-break handovers in urban WiFi networks for moving vehicles, in: 10th Annual Conference on Wireless Ondemand Network Systems and Services, WONS 2013, IEEE, 2013, pp. 170-177.

[11] German Castignani, Alejandro Lampropulos, Alberto Blanc, Nicolas Montavont, Wi2me: a mobile sensing platform for wireless heterogeneous networks, in: 32nd International Conference on Distributed Computing Systems Workshops, ICDCSW 2012, IEEE, 2012, pp. 108-113.

[12] Andrés Arcia-Moret, Laudin Molina, Nicolas Montavont, German Castignani, A. Blanc, Access point discovery in 802.11 networks, in: 6th International Conference on Network Games, Control and Optimization, NetGCooP 2012, Nov. 2014.

[13] Vivek Mhatre, Konstantina Papagiannaki, Using smart triggers for improved user performance in 802.11 wireless networks, in: Proceedings of the 4th International Conference on Mobile Systems, Applications and Services, ACM, 2006, pp. 246-259.

[14] Ali Safa Sadiq, Kamalrulnizam Abu Bakar, Kayhan Zrar Ghafoor, Jaime Lloret SeyedAli Mirjalili, A smart handover prediction system based on curve fitting model for Fast Mobile IPv6 in wireless networks, Int. J. Commun. Syst. 27 (7) (2014) 969-990.

[15] Jungwook Choi, Hyukjoon Lee, Supporting handover in an IEEE 802.11 pbased wireless access system, in: Proceedings of the Seventh ACM International Workshop on VehiculAr InterNETworking, ACM, 2010, pp. 75-80.

[16] Young-uk Chung, Hyukjoon Lee, Yong-Hoon Choi, Chungwon Lee, Proactive caching and forwarding schemes for seamless handover in IEEE WAVE networks, Int. J. Distrib. Sens. Netw. 2013 (2013) 1-16.

[17] Pralhad Deshpande, Anand Kashyap, Chul Sung, Samir R. Das, Predictive methods for improved vehicular WiFi access, in: Proceedings of the 7th International Conference on Mobile Systems, Applications, and Services, ACM, 2009, pp. 263-276.

[18] Daehan Kwak, Jeonghoon Mo, Moonsoo Kang, Investigation of handoffs for IEEE 802.11 networks in vehicular environment, in: First International Conference on Ubiquitous and Future Networks, ICUFN 2009, IEEE, 2009, pp. 89-94.

[19] Julien Montavont, Thomas Noel, IEEE 802.11 handovers assisted by GPS information, in: IEEE International Conference on Wireless and Mobile Computing, Networking and Communications, 2006, WiMob 2006, IEEE, 2006, pp. 166-172.

[20] Daqiang Zhang, Min Chen, Mohsen Guizani, Haoyi Xiong, Mobility prediction in telecom cloud using mobile calls, IEEE Wirel. Commun. 21 (1) (2014) 26-32.

[21] Haitham Abu-Ghazaleh, Attahiru Sule Alfa, Application of mobility prediction in wireless networks using Markov renewal theory, IEEE Trans. Veh. Technol. 59 (2) (2010) 788-802 
[22] Floriano De Rango, Peppino Fazio, Salvatore Marano, Utility-based predictive services for adaptive wireless networks with mobile hosts, IEEE Trans. Veh. Technol. 58 (3) (2009) 1415-1428.

[23] Maximilien Mouton, German Castignani, Raphaël Frank, Thomas Engel, Mroad: scanning-free model-based roaming for 802.11 networks under vehicular mobility, in: Wireless Days (WD), IFIP 2013, IEEE, 2013, pp. 1-8.

[24] Eugenio Giordano, Raphael Frank, Giovanni Pau, Mario Gerla, Corner: a radio propagation model for VANETs in urban scenarios, Proc. IEEE 99 (7) (2011) 1280-1294.

[25] Simulation of urban mobility, [online] accessed January 2015, http://sumo. sourceforge.net/.

[26] Qualnet network simulator, [online] accessed January 2015, http://www. scalable-networks.com.

[27] Eugenio Giordano, Enzo De Sena, Giovanni Pau, Mario Gerla, Vergilius: a scenario generator for VANET, in: IEEE 71st Vehicular Technology Conference, VTC 2010-Spring, IEEE, 2010, pp. 1-5
[28] German Castignani, Juan Monetti, Nicolas Montavont, Andrés Arcia-Moret, Raphaël Frank, Thomas Engel, A study of urban IEEE 802.11 hotspot networks: towards a community access network, in: Wireless Days (WD), 2013 IFIP, IEEE, 2013, pp. 1-8.

[29] German Castignani, Alberto Blanc, Alejandro Lampropulos, Nicolas Montavont, Urban 802.11 community networks for mobile users: current deployments and prospectives, Mob. Netw. Appl. 17 (6) (2012) 796-807.

[30] Javier Gozálvez, Miguel Sepulcre, Ramon Bauza, IEEE 802.11p vehicle to infrastructure communications in urban environments, IEEE Commun. Mag. 50 (5) (2012) 176-183.

[31] K. Paul Yoon, Ching-Lai Hwang, Multiple Attribute Decision Making: An Introduction, Sage Publications, 1995.

[32] Raghu K. Ganti, Fan Ye, Hui Lei, Mobile crowdsensing: current state and future challenges, IEEE Commun. Mag. 49 (11) (2011) 32-39. 